\title{
Holy spirit? An unusual cause of pseudomonal infection in a multiply injured patient
}

\author{
Ian Greaves, K M Porter
}

A 19 year old man jumped from the tenth floor of his residential block of flats in February 1989, sustaining multiple life threatening injuries (table). He was taken to the major injuries unit of this hospital. On arrival, he was resuscitated, intubated, and electively ventilated. A chest drain was inserted; four quadrant peritoneal lavage was clear. Immediate surgical intervention followed (table). He received 53 units of blood and other resuscitation fluid.

After operative intervention the patient developed an episode of septicaemia due to Staphylococcus aureus and Streptococcus pneumoniae; this settled with appropriate intravenous antibiotic therapy including gentamicin. Subsequently he began a steady if unspectacular recovery from his catastrophic injuries. At two weeks he was breathing spontaneously supported by continuous positive airways pressure and at four weeks he was well enough to be interviewed by a psychiatrist. Initial total parenteral nutrition was replaced by feeding through a surgical gastrostomy. After six weeks the halo jacket and external pelvic fixation were removed. Repeated toilet and reconstructive surgery to the leg injuries was performed. Cranial computed tomography revealed small bilateral subdural haematomas, accounting for some fluctuation in mental state.

Suddenly the patient's condition deteriorated. He became confused and feverish, then developed tachypnoea and expectorated green sputum. Signs of pneumonia were seen in the right middle and lower zones of a plain chest radiograph; microbiological investigation showed Pseudomonas aeruginosa to be the causal agent. On the advice of our consultant microbiologist treatment was started with intravenous vancomycin and ciprofloxacin.

We were extremely concerned at the appearance of overwhelming pseudomonal infection on the major injuries unit, and as there were no other patients on the unit we were initially unable to establish a source
Injuries sustained and initial intervention

\begin{tabular}{ll}
\hline Injury & \multicolumn{1}{c}{ Intervention } \\
\hline $\begin{array}{l}\text { Unstable cervical spine injury at C6 } \\
\text { Compound facial skull fracture }\end{array}$ & $\begin{array}{l}\text { Halo traction } \\
\text { Frontal intracranial } \\
\text { pressure bolt inserted }\end{array}$ \\
$\begin{array}{l}\text { Multiple rib fractures } \\
\text { Pneumothorax }\end{array}$ & $\begin{array}{l}\text { Chest drain inserted } \\
\text { Complex pelvic fractures }\end{array}$ \\
$\begin{array}{l}\text { Fracture of right tibial plateau } \\
\text { Bilateral compound lower limb fractures with } \\
\text { almost complete destruction of the left hind } \\
\text { foot }\end{array}$ & $\begin{array}{l}\text { External fixation } \\
\text { No intervention }\end{array}$ \\
& Tracheostomy \\
\hline
\end{tabular}

for this infection. But one of us was present when the patient was visited by his aunt and saw her sprinkle him liberally with holy water. She was immediately stopped, and the remaining water was sent for microbiological investigation. $P$ aeruginosa were found in pure culture and were indistinguishable on detailed phage typing from cultures grown from the patient; there were no other similar isolates from the hospital. Once the situation was explained the aunt immediately stopped sprinkling with holy water.

The patient subsequently recovered rapidly to his state before infection, although he remained a little confused at times. After further surgery he was discharged home almost exactly three months after his admission. Further management consisted of complex bilateral foot surgery to improve both his gait and his appearance. He remains under review and has (reluctantly) received intensive help from psychiatric and rehabilitation services.

We know of no other case of life threatening infection transmitted in this way. We suspect that such transmission is, in fact, more common than realised and may represent a significant source of infection in critically ill patients that will not be noticed unless specifically sought.

\section{A piscatorial epistle}

\section{A J Trevett, D G Lalloo, I H Kevau}

Fish species have been implicated in causing human disease throughout the world in multitudinous ways. In Papua New Guinea there have been cases of scombrotoxic poisoning, ${ }^{12}$ tetrodotoxic poisoning (B Mavo and A J Trevett, unpublished), impalation by garfish and swordfish, ${ }^{34}$ stingray injuries and envenoming, ${ }^{5}$ shark bites, stonefish envenoming, ${ }^{6}$ lion fish envenoming, and impacted fishbones. We are not, however, aware of any reports of respiratory obstruction due to swallowing live fish, and we report two such cases.

\section{Case reports}

Case 1-A 14 year old boy was admitted to Moreguina health centre with a history of acute respiratory difficulty. He had been out spear fishing and had attempted to kill, by biting, a small reef fish. The fish had lodged in his pharynx, and he rapidly became blue and collapsed. On arrival he was apnoeic, pulseless, and cyanosed. A fish tail was seen protruding from his pharynx. Resuscitation was attempted but was unfortunately unsuccessful. A $14 \mathrm{~cm}$ specimen, probably a mado (Atypichthys mado), was later removed from his throat.

Case 2-A 10 year old boy was admitted to Kupiano health centre after swallowing an $8 \mathrm{~cm}$ live reef fish. He had dislodged the fish from a net and attempted to kill it by biting it behind the eye. Unfortunately he had lost hold of the writhing creature, which bolted down his throat. He was understandably distressed and tachypnoeic but not cyanosed. There was no stridor, 